\title{
Continuous Approximation for Interaction Energy Transfer of DNA through Lipid Bilayers
}

\author{
Mansoor H. Alshehri \\ Mathematics Department, College of Science, King Saud University, P.O. Box 2455, Riyadh 11451, Saudi Arabia \\ Correspondence should be addressed to Mansoor H. Alshehri; mhalshehri@ksu.edu.sa
}

Received 4 September 2018; Accepted 11 October 2018; Published 1 November 2018

Academic Editor: Francisco R. Villatoro

Copyright (C) 2018 Mansoor H. Alshehri. This is an open access article distributed under the Creative Commons Attribution License, which permits unrestricted use, distribution, and reproduction in any medium, provided the original work is properly cited.

\begin{abstract}
In this study the interaction energies for single-stranded DNA and double-stranded DNA molecules with a lipid bilayer are investigated. The 6-12 Lennard-Jones potential and continuous approximation are used to derive analytical expressions for these interaction energies. Assuming that there is a circular gap in the lipid bilayer, we determine the relationship of the molecular interaction energy, including the circular gap radius and the perpendicular distance of the single-stranded DNA and doublestranded DNA molecules from the gap. For both single-stranded and double-stranded DNA molecules, the relationship between the minimum energy location and the hole radius $b$ is calculated; in the case of the double-stranded DNA molecule, we assume that the helical phase angle $\phi$ is equal to $\pi$. By minimizing the total interaction energies, the results demonstrate that the singlestranded DNA and double-stranded DNA molecules move through a lipid bilayer when the gap radius $b>10 \AA$ and $b>13.8 \AA$, respectively. The results present in this project can be leveraged to understand the interactions between cell-penetrating peptides and biomembranes, which may improve gene and drug delivery.
\end{abstract}

\section{Introduction}

Recently, nanomaterials have promised an unexpected growth in research and applications in many different areas, due to their nanoscales size, unique features, and distinct properties. Watson and Crick discovered the structure of DNA in 1953, one century after the discovery of the existence of DNA [1]. Owing to the self-assembling, unique physicochemical properties, and the geometric structures of DNA molecules, they have attracted attention as a promising material that has many potential applications in biotechnology and biomedicine such as stochastic biosensors, for the controlled translocation of proteins and as drug carriers across membranes [2-5]. Lipid/DNA complexes hold great promise for future medical applications such as transfect genetic material to the cell core and as novel treatment for various inherited diseases and cancers [6-9]. There are a number of research studies about the interaction behavior of the translocation of molecules into cells, which can help to explain the toxicity of nanoparticles. Maingi et al. use molecular dynamics (MD) simulations to investigate the interactions of a simple DNA nanopore with a lipid bilayer. They demonstrate the close packing of lipids around the stably inserted DNA pore and its cation selectivity [10]. He et al. determined the interaction mechanism between polyarginine peptides and asymmetric membranes by performing a coarse-grained molecular dynamics (CGMD) simulation. Their results show that peptides could be moved through a lipid bilayer by inducing a hydrophilic pore formation in the asymmetric membrane [11, 12]. Khalid et al. utilize (CGMD) simulation to investigate the transfer potential of the DNA through a DPPC/DMTAP bilayer. They also find a high energy barrier to DNA insertion into the bilayer hydrophobic core of the bilayer [13]. In addition, a number of simulations have provided good information about the interactions of DNA and lipids, such as [14-16], where the computational requirements of such a problem did not allow the exploration of the transmigration of the DNA inside the bilayer. Baowan et al. use continuous approximation together with the LennardJones potential function to calculate the interaction energy of silica and carbon nanoparticles with a lipid bilayer [17-19]. 
Alshehri determined the interactions of boron nitride nanotubes as they moved through lipid bilayer membranes using the same technique [20]. Van der Waals energy has played an important role; hence both electrostatic interactions and van der Waals were considered as a part of the computation. In this study, classical applied mathematics is used to calculate the most important interactions of the system and provide the theoretical results which can pave the way toward further improving this area of study. Using continuous approximation and the Lennard-Jones potential function, the molecular interaction energies between ssDNA and dsDNA molecules and the dipalmitoylphosphatidylcholine bilayer (DPPC) are calculated. The penetration behavior of the DNA molecules through an assumed circular gap in the lipid bilayer is investigated. The model formulations for ssDNA and dsDNA molecules and the lipid bilayer are presented in the following section. The mathematical derivations for the ssDNA and dsDNA interaction with the lipid are detailed in Section 3. Moreover, in Section 4 the numerical results are presented, and finally in Section 5 a brief summary of the project is presented.

\section{Modelling Approach}

We determine the molecular interatomic energy between two molecules by employing continuous approximation and the Lennard-Jones potential. We assume that the 6-12 LennardJones potential is expressed as

$$
P(\rho)=-\frac{A}{\rho^{6}}+\frac{B}{\rho^{12}},
$$

where $\rho$ is the distance between two well-defined distinct molecules, $A=4 \varepsilon \sigma^{6}$ and $B=4 \varepsilon \sigma^{12}$ denote the attractive and the repulsive constants, respectively, and $\sigma$ is the interatomic distance when the potential is zero and $\varepsilon$ is the energy well depth. Using a continuous approach, atoms are assumed to be evenly distributed over the molecules and the average atomic densities provided are averaged over its surface, which means that the summation over all atoms involved is replaced by surface integrals. The magnitude of total energy arising from molecular interatomic energy is then obtained mathematically by performing a surface integral over the surface of each molecule, given by

$$
E=\eta_{1} \eta_{2} \int_{S_{1}} \int_{S_{2}} P(\rho) d S_{1} d S_{2}
$$

where $\eta_{1}$ and $\eta_{2}$ denote mean volume densities or the mean surface densities of the atoms on each molecule, and $P(\rho)$ is the potential function for two unbonded atoms. For convenience, we could introduce the integral $I_{n}$ as

$$
I_{n}=\int_{S_{1}} \int_{S_{2}} \rho^{-2 n} d S_{1} d S_{2}, \quad n=3,6
$$

Thus, the total energy can given by

$$
E=\eta_{1} \eta_{2}\left(-A I_{3}+B I_{6}\right) .
$$

At this point, we investigate the energy behavior of the molecular interaction energy of a lipid bilayer and a DNA molecule, by determining the penetration behavior of the ssDNA and dsDNA molecules through an assumed circular gap of radius $b$ in the bilayer. The ssDNA molecule is assumed to be a single helicoid, with a ruled surface having a helix as its boundary. This presumption follows the experimental studies [25-27]. A typical point on the surface of an ssDNA molecule is specified by the coordinates:

$$
\mathbf{H}(\theta, t)=(x, y, z)=\left(r t \cos \theta, r t \sin \theta, \frac{c \theta}{2 \pi}\right),
$$

where $c=34 \AA$ and $r=5.8 \AA$ are the unit cell length and the radius of the ssDNA helix, respectively, and the two parametric variables $\theta$ and $t$ are such that $-\pi<\theta<\pi$ and $0<t<1$. In addition, the dsDNA molecule is modelled as a surface with a double helicoid geometry, and the parametric equation is given by

$$
\begin{aligned}
& \mathbf{G}(\Theta, t)=(x, y, z)=\left(\frac{R}{2}[\cos \Theta+\cos (\Theta-\phi)\right. \\
& +t(\cos \Theta-\cos (\Theta-\phi))], \frac{R}{2}[\sin \Theta+\sin (\Theta-\phi) \\
& \left.\quad+t(\sin \Theta-\sin (\Theta-\phi))], \frac{c \Theta}{2 \pi}\right)
\end{aligned}
$$

where $\phi$ is the helical phase angle parameter, $R=10 \AA$ is the radius of the dsDNA helix, $c=34 \AA$ is the unit cell length of the dsDNA helix, and the parametric variables $\Theta$ and $t$ are such that $-\pi<\Theta<\pi$ and $-1<t<1$. Moreover, with reference to Figure 1 the bilayer of dipalmitoylphosphatidylcholine (DPPC) has been selected as a part of this experiment, each lipid molecule is composed of 12 particles, which are shown in the MARTINI force field as a head group consisting of choline $\left(\mathrm{Q}_{0}\right)$ and phosphate $\left(\mathrm{Q}_{a}\right)$ groups, an intermediate layer consisting of a glycerol group $\left(\mathrm{N}_{a}\right)$, and a carbon tail group $\left(C_{1}\right)$ [23]. We note that the phase behavior of complexes and the considerable diversity in structure stability are depending on the choice of the uncharged lipid and that the chemical type of divalent ion can impact on the stability of the complexes which involves specific interactions [28]. From the work of Bawan et el. [17], the spacing between the two layers of lipids is assumed to be $3.36 \AA$, and the initial value for the area per lipid is assumed to be $65 \AA^{2}$, which is taken from the work of Berger et al. [29]. The head and tail groups could be modelled as infinite boxes of thicknesses 4 and 15, respectively, following the work of Bawan et el. [18].

Using the rectangular Cartesian coordinate system $(x, y$, $z$ ), the six layers of the lipid bilayer are assumed to have the coordinates $(a \cos \vartheta, a \cos \vartheta, z)$ and $(a \cos \vartheta, a \cos \vartheta, z-$ 37.36) for the upper and lower head groups, respectively. By assuming that the surface of the upper head group is located on the $x y$-plane, $z=0$, the coordinates of a typical point on this plane can be given by $(a \cos \vartheta, a \cos \vartheta, 0)$, where $b<a<$ $\infty$ and $0<\vartheta<2 \pi$. In addition, the upper and lower intermediate layers have coordinates $(a \cos \vartheta, a \cos \vartheta, z-4)$ and ( $a \cos \vartheta, a \cos \vartheta, z-37.36$ ), respectively, as well as the upper 


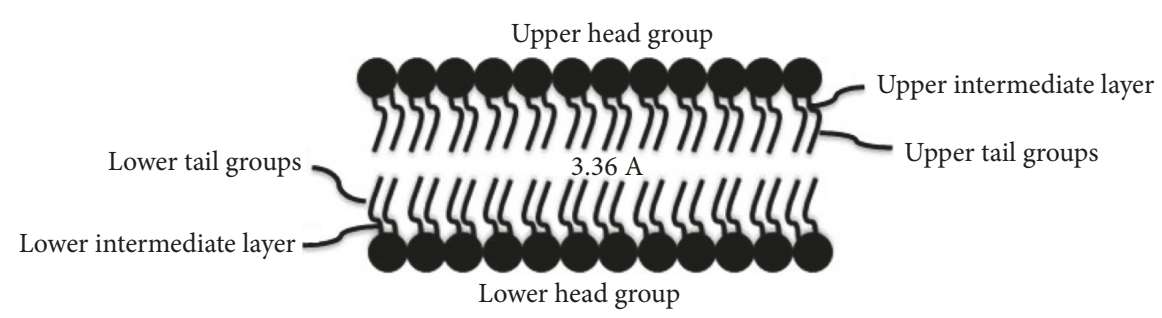

FIGURE 1: Schematic of the lipid bilayer.

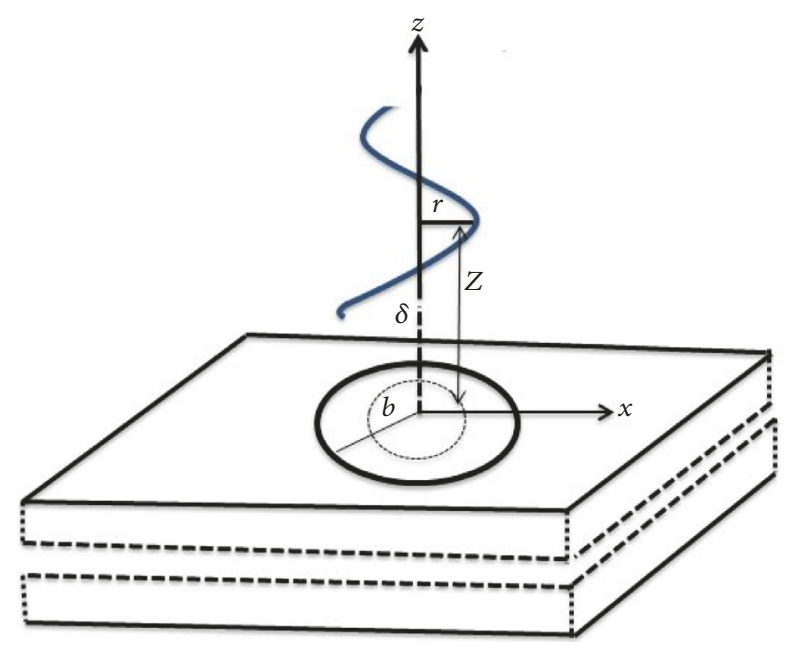

Figure 2: An ssDNA molecule penetrating a lipid bilayer gap.

and lower tail groups $(a \cos \vartheta, a \cos \vartheta, z-4)$ and $(a \cos \vartheta$, $a \cos \vartheta, z-22.36)$, respectively.

\section{Mathematical Derivation}

In this study, the techniques of volume and surface integration are used to compute the total interaction energy of the ssDNA and dsDNA molecules with a lipid bilayer. The head group and the tail group are modelled as rectangular boxes, and the intermediate group is assumed to be a flat plane. Moreover, the bilayer is assumed to stay in an equilibrium structure while interacting with the DNA molecule; thus the interaction between the two layers could be ignored, and the total interaction energy of the system is assumed to contain

(i) The interactions between the volumes of the upper and lower head groups and the surfaces of the ssDNA and dsDNA molecules

(ii) The interactions between the surfaces of the upper and lower intermediate layers and the surfaces of the ssDNA and dsDNA molecules

(iii) The interactions between the volumes of the upper and lower tail groups and the surfaces of the ssDNA and dsDNA molecules

In the following subsections, the mathematical derivations of the interactions of this system are detailed.
3.1. Interaction Energy of ssDNA Penetrating a Lipid Bilayer Hole. A unit cell of the ssDNA molecule of radius $r$ is examined at a distance $Z$ above a gap of radius $b>r$ in the lipid bilayer, where $Z$ is the perpendicular distance from the gap of the uppermost surface of the lipid bilayer to the center of the ssDNA molecule, as shown in Figure 2. Thus, a typical surface element of the ssDNA molecule has the coordinates given by $(r t \cos \theta, r t \sin \theta, c \theta / 2 \pi+Z)$, and without loss of generality the coordinates of a typical point on the ssDNA molecule can be given by $(\alpha, 0, Z+c \theta / 2 \pi)$, where $\alpha=r t$. Firstly, the interaction energy between an ssDNA molecule and a flat plane, which has the coordinates $(a \cos \vartheta, a \cos \vartheta, 0)$, is calculated. Therefore, the distance of the surface of an ssDNA to the plane is given by

$$
\begin{aligned}
\rho^{2} & =(\alpha-a \cos \vartheta)^{2}+(-a \cos \vartheta)^{2}+\left(Z+\frac{c \theta}{2 \pi}\right)^{2} \\
& =\alpha^{2}-2 \alpha \cos \vartheta+a^{2}+\left(Z+\frac{c \theta}{2 \pi}\right)^{2} \\
& =(\alpha-a)^{2}+4 a \alpha \sin ^{2}\left(\frac{\vartheta}{2}\right)+\left(Z+\frac{c \theta}{2 \pi}\right)^{2}
\end{aligned}
$$

and from (3) the integral $I_{n}$ can be written as 


$$
I_{n}^{\mathrm{p}}=\int_{-\pi}^{\pi} \int_{-\pi}^{\pi} \int_{0}^{1} \int_{b}^{\infty} a\left[(\alpha-a)^{2}+4 a \alpha \sin ^{2}\left(\frac{\vartheta}{2}\right)+\left(Z+\frac{c \theta}{2 \pi}\right)^{2}\right]^{-n} \times\left(1+\frac{4 r^{2} \pi^{2}}{c^{2}} t^{2}\right)^{1 / 2} d a d t d \theta d \vartheta
$$

where $\mathrm{p}$ is referred to in the plane interaction. By setting $\xi=$ $(\alpha-a)^{2}+(Z+c \theta / 2 \pi)^{2}$ and $\beta=4 a \alpha$ and defining the integral $K_{n}$ as

$$
K_{n}=\int_{-\pi}^{\pi}\left[\xi+\beta \sin ^{2}\left(\frac{\vartheta}{2}\right)\right]^{-n} d \vartheta
$$

upon making the substitution $u=\sin ^{2}(\vartheta / 2) \Longrightarrow d \vartheta=$ $u^{-1 / 2}(1-u)^{-1 / 2} d u$, the integral $K_{n}$ becomes

$$
\begin{aligned}
K_{n} & =2 \int_{0}^{1}[\xi+\beta u]^{-n} u^{-1 / 2}(1-u)^{-1 / 2} d u \\
& =2 \xi^{-n} \int_{0}^{1}\left[1+\left(\frac{\beta}{\xi}\right) u\right]^{-n} u^{-1 / 2}(1-u)^{-1 / 2} d u \\
& =2 \pi \xi^{-n} F\left(n, \frac{1}{2} ; 1 ;-\frac{\beta}{\xi}\right)
\end{aligned}
$$

$$
\begin{aligned}
I_{n}^{\mathrm{p}} & =2 \pi \sum_{k=0}^{n-1} \frac{(4 r)^{k}(1-n)_{k}(1 / 2)_{k}}{(k !)^{2}} \\
& \cdot \int_{-\pi}^{\pi} \int_{0}^{1} \int_{b}^{\infty} \frac{a^{k+1} t^{k}}{\left[(r t-a)^{2}+(Z+c \theta / 2 \pi)^{2}\right]^{n-1 / 2}\left[(r t+a)^{2}+(Z+c \theta / 2 \pi)^{2}\right]^{k}} \times\left(1+\frac{4 r^{2} \pi^{2}}{c^{2}} t^{2}\right)^{1 / 2} d a d t d \theta .
\end{aligned}
$$

The above integrals become extremely complex; therefore we leverage a standard integration package, such as MAPLE, to evaluate them numerically. Thus, the total interaction energy between the ssDNA molecule and the intermediate layers could be given by

$$
\begin{aligned}
I_{n}^{\mathrm{b}} & =2 \pi \sum_{k=0}^{n-1} \frac{(4 r)^{k}(1-n)_{k}(1 / 2)_{k}}{(k !)^{2}} \\
& \cdot \int_{-l}^{0} \int_{-\pi}^{\pi} \int_{0}^{1} \int_{b}^{\infty} \frac{a^{k+1} t^{k}}{\left[(r t-a)^{2}+(Z+c \theta / 2 \pi-z)^{2}\right]^{n-1 / 2}\left[(r t+a)^{2}+(Z+c \theta / 2 \pi-z)^{2}\right]^{k}} \times\left(1+\frac{4 r^{2} \pi^{2}}{c^{2}} t^{2}\right)^{1 / 2} d a d t d \theta d z
\end{aligned}
$$

Furthermore, the interaction between the ssDNA molecule and a box which is assumed to have a thickness of $l$, where $-l<z<0$, can be identified by following similar steps undertaken with the term $(Z+c \theta / 2 \pi)$ replaced by $(Z+c \theta / 2 \pi-z)$; thus the integral $I_{n}$ which is given in (3) becomes where $b$ denotes the box interaction, and the thickness of the lipid bilayer head group is $l=4 \AA$, and $l=15$ for that of the tail group. Thus, the total interaction energy between the ssDNA molecule and the head or the tail group is given by

$$
E_{\mathrm{ssDNA}}^{\mathrm{b}}=\eta_{i} \eta_{d}\left(-A I_{3}^{\mathrm{b}}+B I_{6}^{\mathrm{b}}\right) \text {, }
$$

where $i$ can be either $H$ or $T$. where $F\left(a^{*}, b^{*} ; c^{*} ; z^{*}\right)$ is the standard hypergeometric function [30]. Moreover, by using a Pfaff transformation [30], $K_{n}$

$$
\begin{aligned}
K_{n} & =2 \pi \xi^{-n}\left(\frac{\xi}{\xi+\beta}\right)^{1 / 2} F\left(1-n, \frac{1}{2} ; 1 ; \frac{\beta}{\xi+\beta}\right) \\
& =2 \pi \xi^{-n}\left(\frac{\xi}{\xi+\beta}\right)^{1 / 2} \sum_{k=0}^{n-1} \frac{(1-n)_{k}(1 / 2)_{k}}{(k !)^{2}}\left(\frac{\beta}{\xi+\beta}\right)^{k} \\
& =2 \pi\left[(r t-a)^{2}+\left(Z+\frac{c \theta}{2 \pi}\right)^{2}\right]^{-n+1 / 2} \\
& \cdot \sum_{k=0}^{n-1} \frac{(4 a r t)^{k}(1-n)_{k}(1 / 2)_{k}}{(k !)^{2}\left[(r t+a)^{2}+(Z+c \theta / 2 \pi)^{2}\right]^{k}} .
\end{aligned}
$$

3.2. Interaction Energy of dsDNA Penetrating a Lipid Bilayer Gap. In this subsection, the energy behavior of a unit of the dsDNA molecule is considered. With reference to Figure 3, the dsDNA molecule is assumed to be located on the $z$-axis at a distance $Z$ above the gap in the lipid bilayer. A typical point on the dsDNA has the following coordinates: 


$$
\begin{aligned}
& \left(\frac{R}{2}[\cos \Theta+\cos (\Theta-\phi)+t(\cos \Theta-\cos (\Theta-\phi))],\right. \\
& \frac{R}{2}[\sin \Theta+\sin (\Theta-\phi)+t(\sin \Theta-\sin (\Theta-\phi))], \\
& \left.\quad \frac{c \Theta}{2 \pi}+Z\right) .
\end{aligned}
$$

Here, the helical phase angle $\phi$ is assumed to be equal to $\pi(\phi=\pi)$, which is close to the physical value of the helical angle which is equal to $12 \pi / 17$ ( $\phi=12 \pi / 17)$. We comment that this assumption can lead to a substantial simplification in the analytical expression for the interactions. Thus, the parametric equation of a typical surface element of the dsDNA molecule interacting with the lipid bilayer is given by ( $R t \cos \Theta, R t \sin \Theta, c \Theta / 2 \pi+Z$ ). As before, without loss of generality, the coordinates of a typical point on the dsDNA molecule can be given by $(\lambda, 0, Z+c \Theta / 2 \pi)$, where $\lambda=R t$. Thus, the distance between two typical points on the surface of a dsDNA and the plane surface is given by

$$
\rho^{2}=(\lambda-a)^{2}+4 a \lambda \sin ^{2}\left(\frac{\vartheta}{2}\right)+\left(Z+\frac{c \Theta}{2 \pi}\right)^{2},
$$

and the total interaction energy between the dsDNA molecule and the intermediate layers can be given by

$$
E_{\mathrm{dsDNA}}^{\mathrm{P}}=\eta_{I} \eta_{D}\left(-A I_{3}^{\mathrm{P}}+B I_{6}^{\mathrm{P}}\right),
$$

where

$$
\begin{aligned}
I_{n}^{\mathrm{P}} & =2 \pi \sum_{k=0}^{n-1} \frac{(4 R)^{k}(1-n)_{k}(1 / 2)_{k}}{(k !)^{2}} \\
& \cdot \int_{-\pi}^{\pi} \int_{-1}^{1} \int_{b}^{\infty} \frac{a^{k+1} t^{k}}{\left[(R t-a)^{2}+(Z+c \Theta / 2 \pi)^{2}\right]^{n-1 / 2}\left[(R t+a)^{2}+(Z+c \Theta / 2 \pi)^{2}\right]^{k}} \times\left(1+\frac{4 R^{2} \pi^{2}}{c^{2}} t^{2}\right)^{1 / 2} d a d t d \Theta .
\end{aligned}
$$

Moreover, the total energy of the interaction of a dsDNA molecule with the head or the tail group is given by

$$
E_{\mathrm{dsDNA}}^{\mathrm{B}}=\eta_{i} \eta_{D}\left(-A I_{3}^{\mathrm{B}}+B I_{6}^{\mathrm{B}}\right),
$$

where $i$ can be either $H$ or $T$, and

$$
\begin{aligned}
I_{n}^{\mathrm{B}} & =2 \pi \sum_{k=0}^{n-1} \frac{(4 R)^{k}(1-n)_{k}(1 / 2)_{k}}{(k !)^{2}} \\
& \cdot \int_{-l}^{0} \int_{-\pi}^{\pi} \int_{-1}^{1} \int_{b}^{\infty} \frac{a^{k+1} t^{k}}{\left[(R t-a)^{2}+(Z+c \Theta / 2 \pi-z)^{2}\right]^{n-1 / 2}\left[(R t+a)^{2}+(Z+c \Theta / 2 \pi-z)^{2}\right]^{k}} \times\left(1+\frac{4 R^{2} \pi^{2}}{c^{2}} t^{2}\right)^{1 / 2} d a d t d \Theta d z .
\end{aligned}
$$

\section{Numerical Results and Discussions}

As a part of the experiment, the interaction energies for the ssDNA and dsDNA molecules and the lipid bilayer are calculated and for the dsDNA we assume that the helical phase angle $\phi$ is equal to $\pi$. To obtain the numerical results of the above systems, we use the algebraic computer package MAPLE together with the parameter values given in Tables 1 and 2. Firstly, we show the equilibrium positions with distance $\delta$ for the ssDNA and dsDNA molecules assuming that the DNA molecules are interacting with a lipid bilayer without a gap. Graphically in Figure 4 the relationship between the interaction energy and the distances $\delta$ is shown, where $\delta$ is referred to as the closest distance from the upper lipid head group to the DNA molecule and $(\delta=Z-17)$. The results demonstrate that for both the ssDNA and dsDNA molecules the equilibrium distances $\delta$ are $\approx 3.60 \AA$ above the bilayer. In addition, due to the difference in the number of atoms between the ssDNA and dsDNA molecules, it can be seen that the value of the energy of the ssDNA molecule is less than the energy value of the dsDNA molecule. In addition, Figure 5 shows the relationship between the total interaction energy and the perpendicular distance $Z$ for various values of the gap radius in the lipid bilayer. Results indicate that when the values of the gap radius of the lipid bilayer are bigger than $\approx 10.5 \AA$, the DNA molecule can penetrate the gap. In addition, the minimum energy occurs when the radius of the gap is equal to $10.5 \AA$. Moreover, Figure 6 shows that the dsDNA molecule can be moved through the gap radius when the value of the radius is larger than $\approx 14 \AA$. The optimal value of the radius for a dsDNA molecule which is provided with the minimum energy to penetrate the lipid bilayer is approximately $14.1 \AA$. Furthermore, for both the ssDNA and dsDNA molecules when the radius of the gap becomes larger, the perpendicular distance $Z$ to the upper head group of the lipid bilayer becomes smaller. In addition, the results demonstrate that the DNA molecule requires a large amount of energy to move inside the bilayer. These results are in agreement with work of Khalid et al. [13]. 
TABLE 1: Numerical values for constants used in this paper ( $*$ denotes data from [21], ** denotes data from [22], and $* * *$ denotes data from $[18])$.

\begin{tabular}{lc}
\hline Constant & Value \\
\hline$*$ Radius of dsDNA & $R=10 \AA$ \\
$*$ Length of DNA & $c=34 \AA$ \\
$* *$ Radius of ssDNA & $r=5.4 \AA$ \\
$* * *$ Atomic density of the head group & $\eta_{H}=0.0077 \AA^{-3}$ \\
$* * *$ Atomic density of the intermediate group & $\eta_{I}=0.0308 \AA^{-2}$ \\
$* * *$ Atomic density of the tail group & $\eta_{T}=0.0082 \AA^{-3}$ \\
Atomic density of ssDNA & $\eta_{d}=0.80 \AA^{-2}$ \\
Atomic density of dsDNA & $\eta_{D}=0.84 \AA^{-2}$ \\
\hline
\end{tabular}

TABLE 2: Lennard-Jones parameters for DPPC lipid interacting with DNA [23, 24].

\begin{tabular}{lcccc}
\hline Interaction & $\varepsilon(\mathrm{kcal} / \mathrm{mol})$ & $\sigma(\AA)$ & $\mathrm{A}\left(\AA^{6} \mathrm{kcal} / \mathrm{mol}\right)$ & $\mathrm{B}\left(\AA^{12} \mathrm{kcal} / \mathrm{mol}\right)$ \\
\hline Head group & 0.2718 & 4.7996 & 13540.92566 & 201188130.2 \\
Intermediate layer & 0.2567 & 4.04596 & 4989.875741 & 26282244.42 \\
Tail group & 0.2396 & 4.04596 & 4657.867035 & 24533516.72 \\
\hline
\end{tabular}

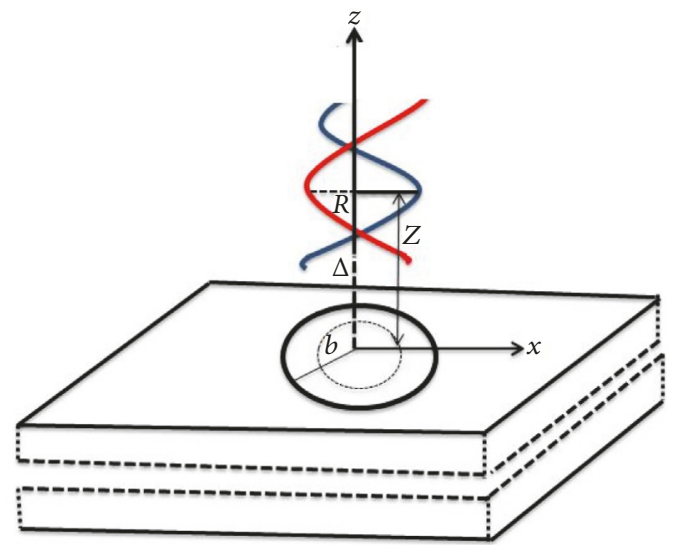

Figure 3: A dsDNA molecule penetrating a hole lipid bilayer.

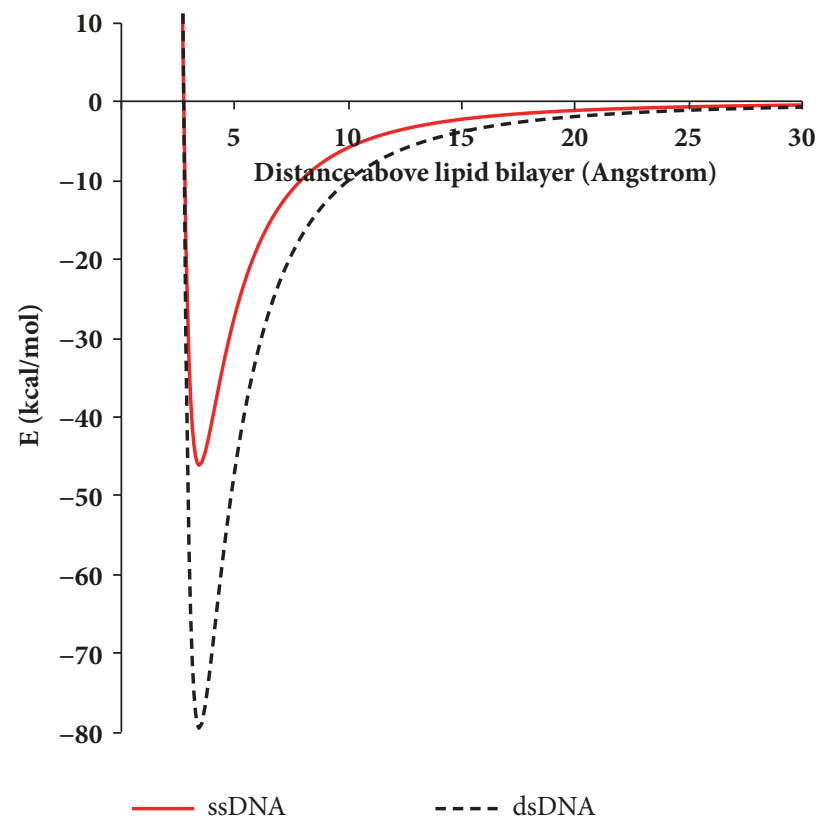

FIGURE 4: Energy profiles for ssDNA and dsDNA interacting with a lipid bilayer without a gap. 


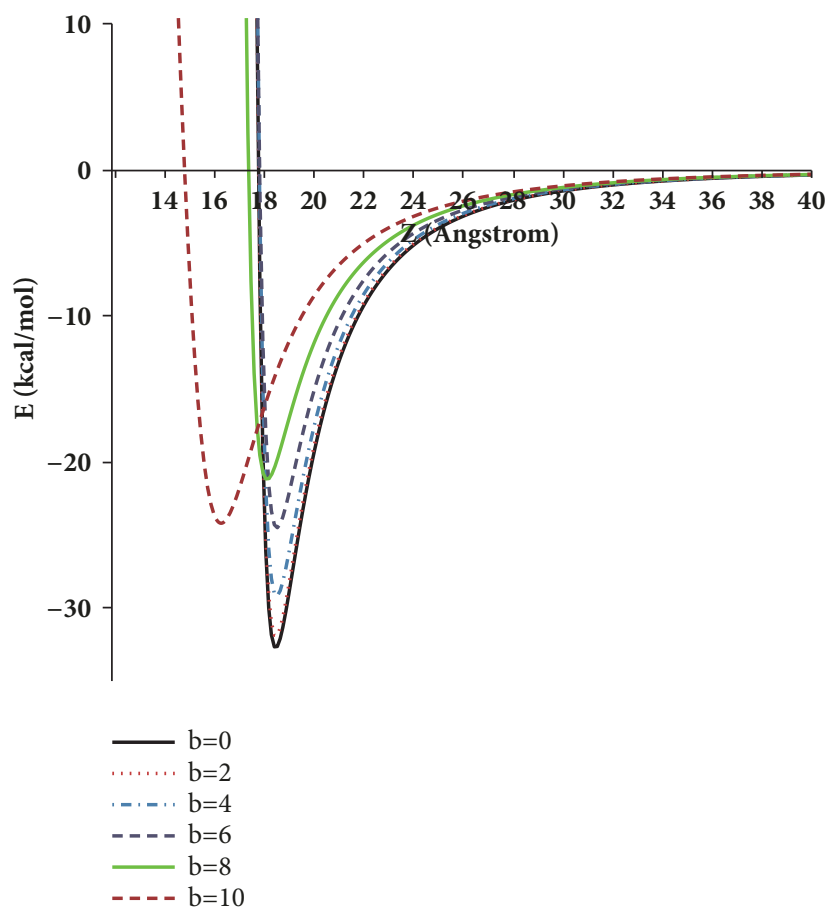

(a) Gap radius $b=0,2,4,6,8$, and $10 \AA$

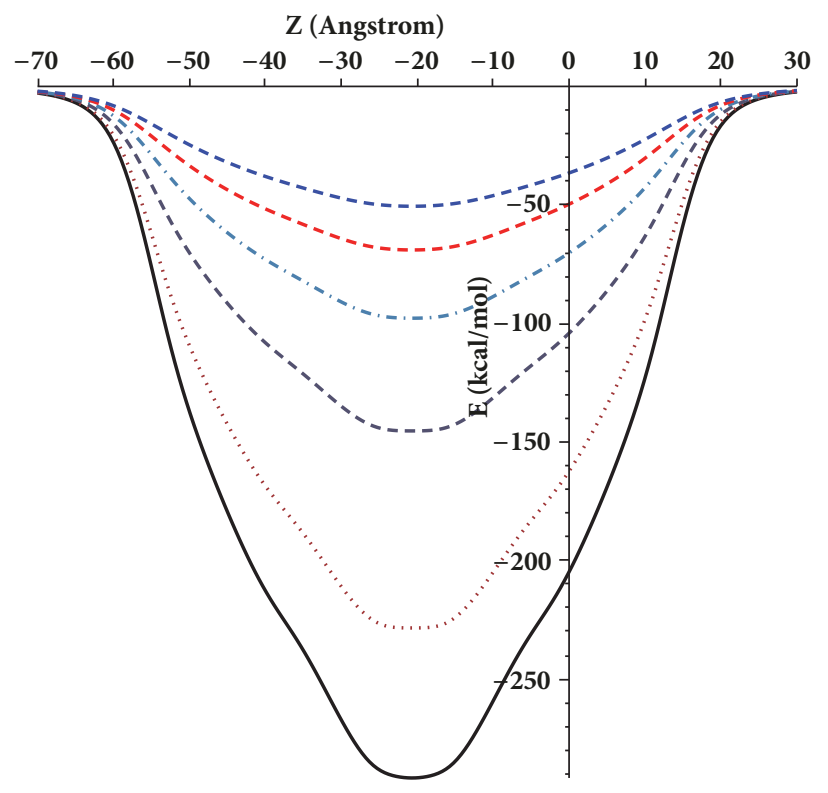

$$
\begin{array}{ll}
- & b=10.5 \\
\cdots \cdots & b=11 \\
--- & b=12 \\
-\cdots & b=13 \\
--- & b=14 \\
--- & b=15
\end{array}
$$

(b) Gap radius $b=10.5-15 \AA$

FIgURE 5: Total energy for ssDNA molecule interacting with different values of the gap radius $b$ with respect to the perpendicular distance $Z$. 


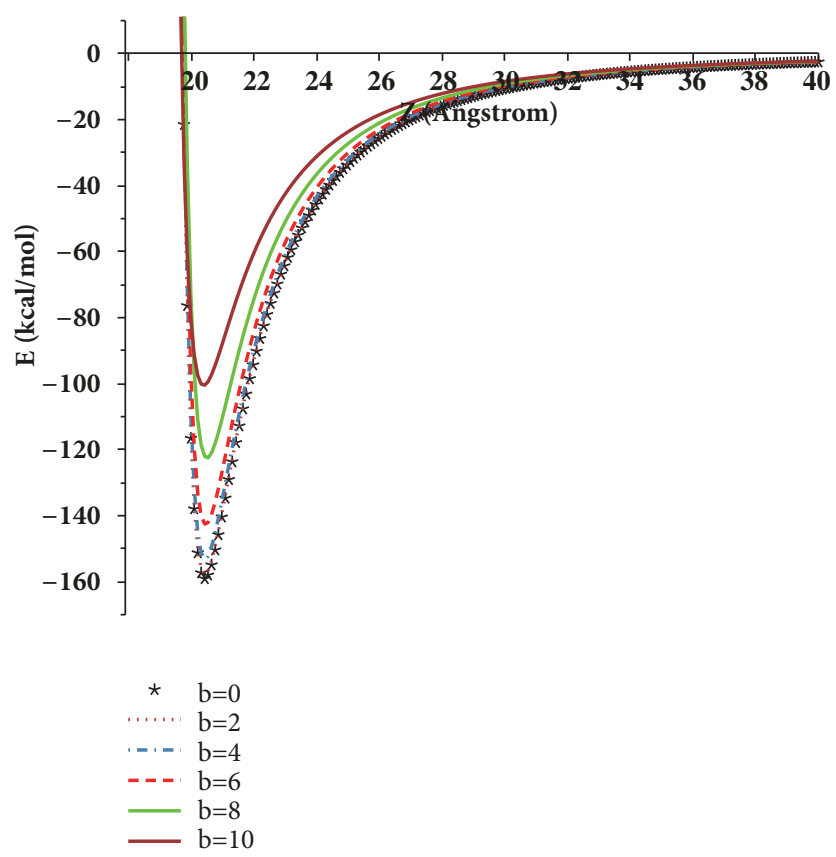

(a) Gap radius $b=0,2,4,6,8$, and $10 \AA$

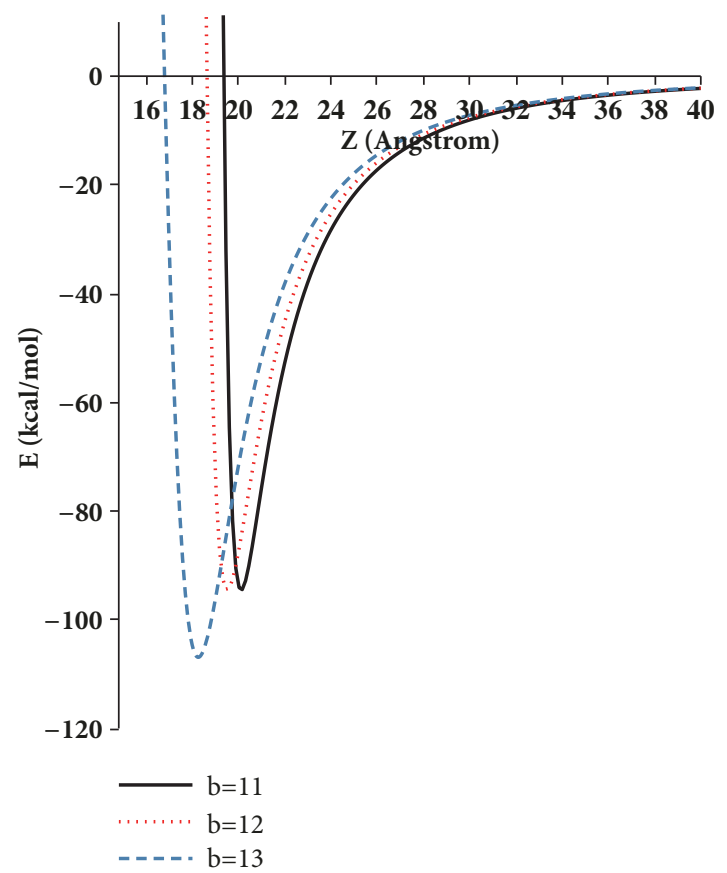

(b) Gap radius $b=11,12$, and $13 \AA$

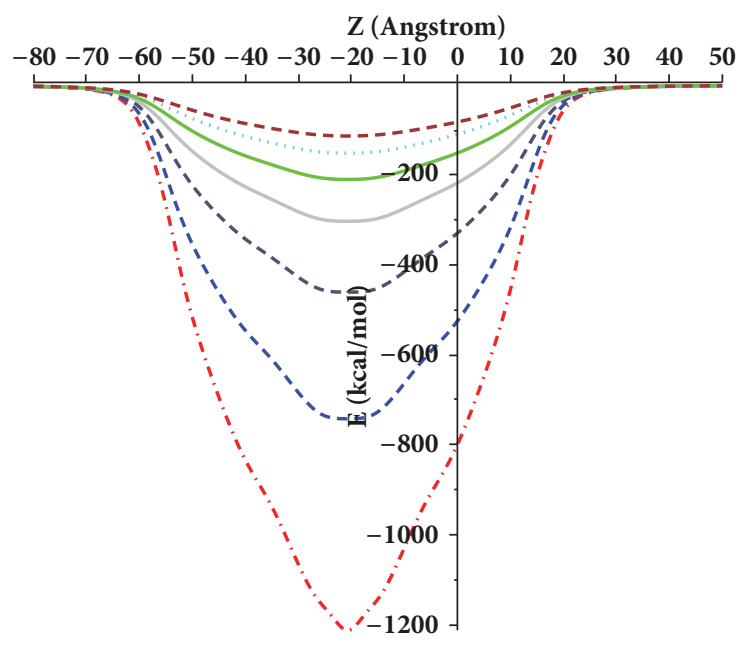

$$
\begin{aligned}
\ldots-\cdots & b=14 \\
-\cdots & b=15 \\
-\cdots & b=16 \\
- & b=17 \\
- & b=18 \\
\cdots \cdots & b=19 \\
-\cdots & b=20
\end{aligned}
$$

(c) Gap radius $b=14-20 \AA$

Figure 6: Total energy for dsDNA molecule interacting with different values of the gap radius $b$ with respect to the perpendicular distance $Z$. 


\section{Conclusion}

This study introduces a mathematical modelling approach which is used to adopt the Lennard-Jones potential together with continuous approximation to investigate the interaction energy of single-stranded and double-stranded DNA molecules penetrating into a DPPC lipid bilayer. The numerical results were obtained by employing the algebraic computer package MAPLE. In the absence of a gap in the lipid, the distance from the edge of the ssDNA and dsDNA molecules to the upper head group is obtained where the minimum energy occurs. The results indicate that for both types of molecules the spacing is $\approx 3.60 \AA$. Moreover, the energy behavior for both the ssDNA and dsDNA molecules is measured while penetrating a presumably circular gap on the infinite plane of a lipid bilayer. Results show that an ssDNA molecule is moved inside the bilayer when the gap radius is larger than $10 \AA$. In addition, a dsDNA molecule can be located inside the bilayer when the radius of the bilayer gap is larger than $13.8 \AA$. In addition, the results demonstrate that the potential energy required for both the ssDNA and dsDNA molecules to penetrate the lipid bilayer is high. These results compare favourably with other methods, such as the experiments and (MD) simulations, conducted by $[10,13]$. Our study can provide insights into the molecular interactions between a DNA molecule and a lipid bilayer. Therefore, it can also provide direction for targeted drug and gene delivery. In addition, this study can provide additional clarification as to the interaction of DNA with lipids and of the mechanisms by which DNA might move through cells.

\section{Data Availability}

No data have been used to support this study.

\section{Conflicts of Interest}

The author declares that they have no conflicts of interest.

\section{Acknowledgments}

This project was supported by King Saud University, Deanship of Scientific Research, College of Science Research Center.

\section{References}

[1] J. D. Watson and F. H. C. Crick, "Molecular structure of nucleic acids: a structure for deoxyribose nucleic acid," Nature, vol. 171, no. 4356, pp. 737-738, 1953.

[2] D. H. Stoloff and M. Wanunu, "Recent trends in nanopores for biotechnology," Current Opinion in Biotechnology, vol. 24, no. 4, pp. 699-704, 2013.

[3] S. Howorka, S. Cheley, and H. Bayley, "Sequence-specific detection of individual DNA strands using engineered nanopores," Nature Biotechnology, vol. 19, no. 7, pp. 636-639, 2001.

[4] J. Li, H. T. Ng, A. Cassell et al., "Carbon nanotube nanoelectrode array for ultrasensitive DNA detection," Nano Letters, vol. 3, no. 5, pp. 597-602, 2003.
[5] D. M. Copolovici, K. Langel, E. Eriste, and Ü. Langel, "Cellpenetrating peptides: design, synthesis, and applications," ACS Nano, vol. 8, no. 3, pp. 1972-1994, 2014.

[6] P. L. Felgner and G. Rhodes, "Gene therapeutics," Nature, vol. 349, no. 6307, pp. 351-352, 1991.

[7] "Gene Therap," in Therapeutic Mechanisms and Strategies, pp. 965-966, Marcel Dekker, New York, NY, USA, 2000.

[8] H. F. Willard, "Artificial chromosomes coming to life," Science, vol. 290, no. 5495, pp. 1308-1309, 2000.

[9] K. Ewert, N. L. Slack, A. Ahmad et al., "Cationic lipid-DNA complexes for gene therapy: understanding the relationship between complex structure and gene delivery pathways at the molecular level," Current Medicinal Chemistry, vol. 11, no. 2, pp. 133-149, 2004.

[10] V. Maingi, J. R. Burns, J. J. Uusitalo, S. Howorka, S. J. Marrink, and M. S. P. Sansom, "Stability and dynamics of membranespanning DNA nanopores," Nature Communications, vol. 8, pp. $1-12,2017$.

[11] X. He, M. Lin, B. Sha et al., "Coarse-grained molecular dynamics studies of the translocation mechanism of polyarginines across asymmetric membrane under tension," Scientific Reports, vol. 5, no. 1, pp. 1-10, 2015.

[12] X. C. He, Z. G. Qu, and F. Xu, "Simulation study of interaction mechanism between peptide and asymmetric membrane," Molecular Simulation, vol. 43, no. 1, pp. 34-41, 2017.

[13] S. Khalid, P. J. Bond, J. Holyoake, R. W. Hawtin, and M. S. P. Sansom, "DNA and lipid bilayers: Self-assembly and insertion," Journal of the Royal Society Interface, vol. 5, no. 3, pp. S241-S250, 2008.

[14] S. Bandyopadhyay, M. Tarek, and M. L. Klein, "Molecular dynamics study of a lipid-DNA complex," The Journal of Physical Chemistry B, vol. 103, no. 46, pp. 10075-10080, 1999.

[15] O. Farago, N. Grønbech-Jensen, and P. Pincus, "Mesoscale Computer Modeling of Lipid-DNA Complexes for Gene Therapy," Physical Review Letters, vol. 96, no. 1, 2006.

[16] M. Tarek, "Membrane electroporation: A molecular dynamics simulation," Biophysical Journal, vol. 88, no. 6, pp. 4045-4053, 2005.

[17] D. Baowan, B. J. Cox, and J. M. Hill, "Instability of C60 fullerene interacting with lipid bilayer," Journal of Molecular Modeling, vol. 18, no. 2, pp. 549-557, 2012.

[18] D. Baowan, B. J. Cox, and J. M. Hill, "Instability of carbon nanoparticles interacting with lipid bilayers," RSC Advances, vol. 5, no. 8, pp. 5508-5515, 2015.

[19] D. Baowan, H. Peuschel, A. Kraegeloh, and V. Helms, "Energetics of liposomes encapsulating silica nanoparticles," Journal of Molecular Modeling, vol. 19, no. 6, pp. 2459-2472, 2013.

[20] M. H. Alshehri, "Interactions of boron nitride nanotubes with lipid bilayer membranes," Journal of Computational and Theoretical Nanoscience, vol. 15, no. 1, pp. 311-316, 2018.

[21] B. Alberts, A. Johnson, J. Lewis, M. Raff, K. Roberts, and P. Walter, "Molecular Biology of the Cell," in Garland Science, New York, NY, USA, 2008.

[22] H. Gao, Y. Kong, D. Cui, and C. S. Ozkan, "Spontaneous insertion of DNA oligonucleotides into carbon nanotubes," Nano Letters, vol. 3, no. 4, pp. 471-473, 2003.

[23] S. J. Marrink, H. J. Risselada, S. Yefimov, D. P. Tieleman, and A. H. De Vries, "The MARTINI force field: coarse grained model for biomolecular simulations," The Journal of Physical Chemistry $B$, vol. 111, no. 27, pp. 7812-7824, 2007. 
[24] S. L. Mayo, B. D. Olafson, and W. A. Goddard III, "DREIDING: a generic force field for molecular simulations," The Journal of Physical Chemistry C, vol. 94, no. 26, pp. 8897-8909, 1990.

[25] K. E. Duderstadt, K. Chuang, and J. M. Berger, "DNA stretching by bacterial initiators promotes replication origin opening," Nature, vol. 478, no. 7368, pp. 209-213, 2011.

[26] V. Luzzati, A. Mathis, F. Masson, and J. Witz, "Structure transitions observed in DNA and poly A in solution as a function of temperature and $\mathrm{pH}$," Journal of Molecular Biology, vol. 10, no. 1, pp. 28-41, 1964.

[27] B. Hingerty and S. Broyde, "Helix geometry of single stranded DNA 'A' and 'B' forms from minimum energy conformations of dimeric subunits," Nucleic Acids Research, vol. 5, no. 1, pp. 127137, 1978.

[28] D. H. Mengistu, K. Bohinc, and S. May, "Binding of DNA to zwitterionic lipid layers mediated by divalent cations," The Journal of Physical Chemistry B, vol. 113, no. 36, pp. 12277-12282, 2009.

[29] O. Berger, O. Edholm, and F. Jähnig, "Molecular dynamics simulations of a fluid bilayer of dipalmitoylphosphatidylcholine at full hydration, constant pressure, and constant temperature," Biophysical Journal, vol. 72, no. 5, pp. 2002-2013, 1997.

[30] I. S. Gradshteyn and I. M. Ryzhik, Tables of Integrals, Series and Products Academic, New York, 1980. 


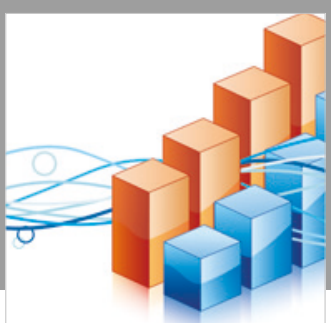

Advances in

Operations Research

\section{-n-m}
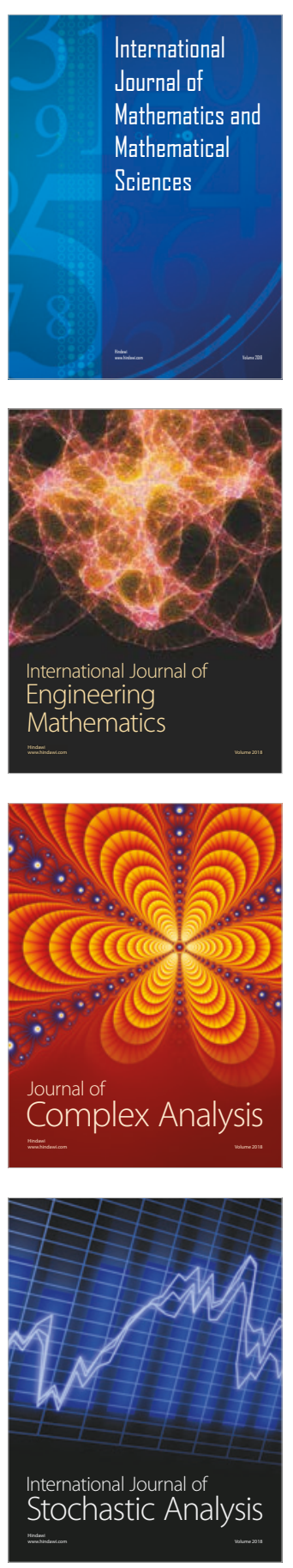
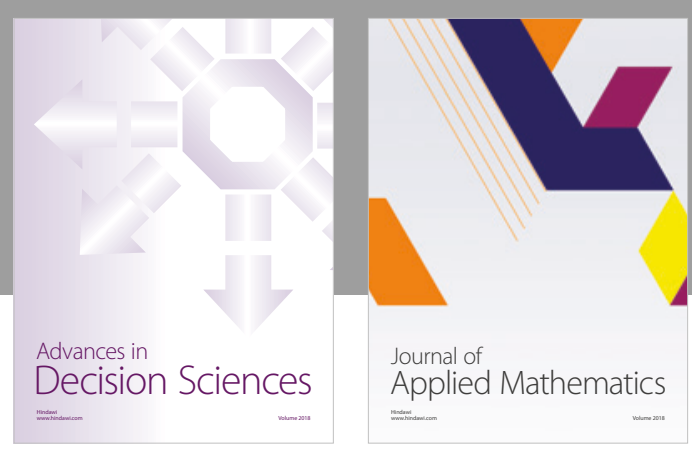

Journal of

Applied Mathematics
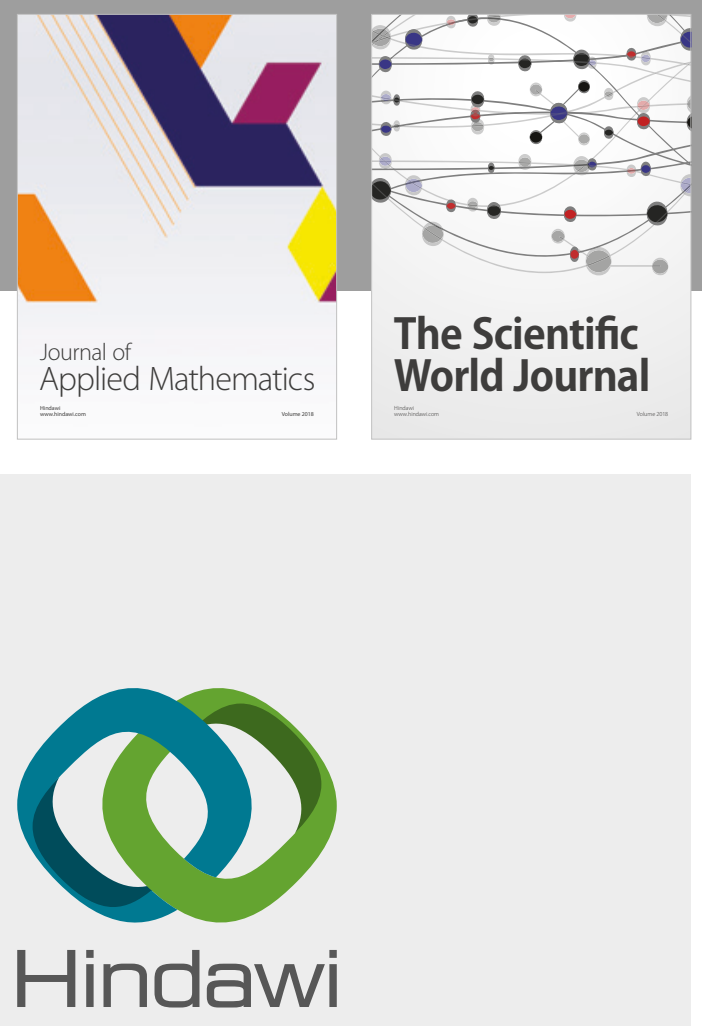

Submit your manuscripts at

www.hindawi.com

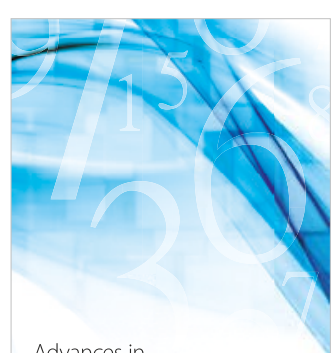

Advances in
Numerical Analysis
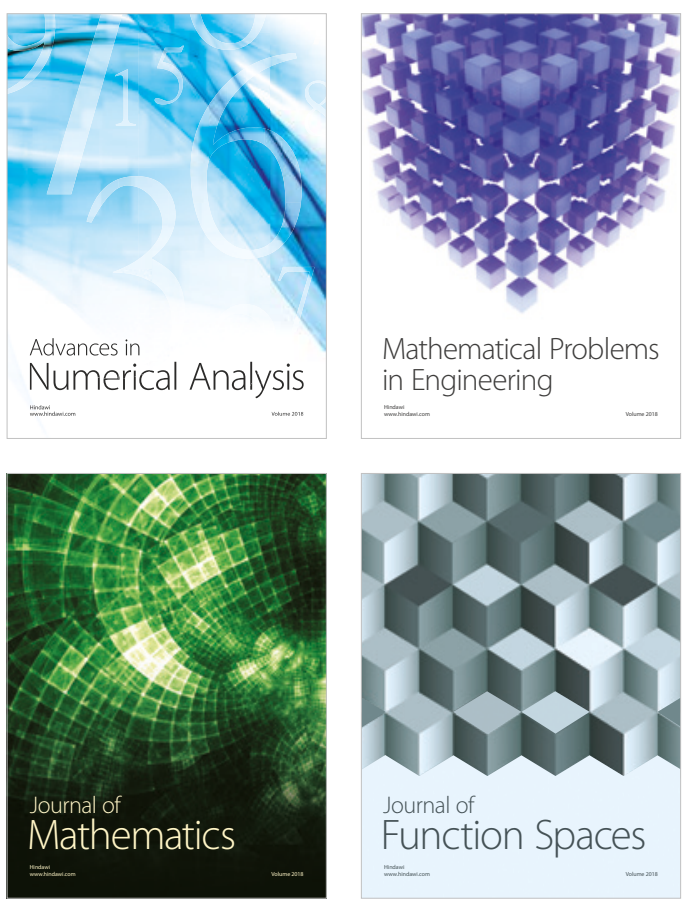

Mathematical Problems in Engineering

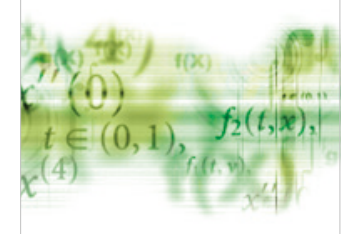

International Journal of

Differential Equations

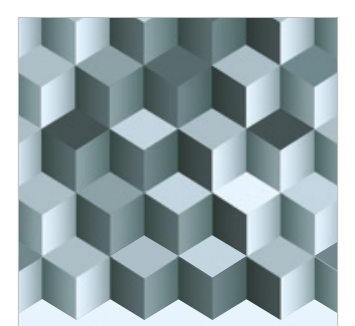

Journal of

Function Spaces

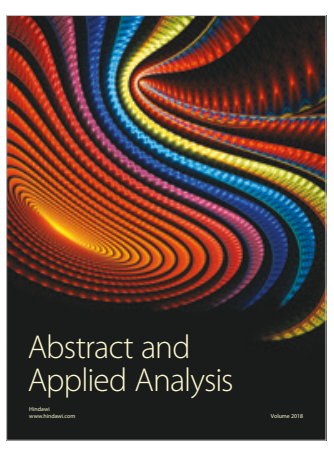

The Scientific

World Journal

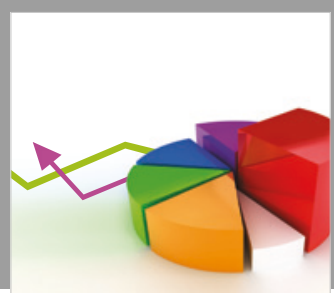

Journal of

Probability and Statistics
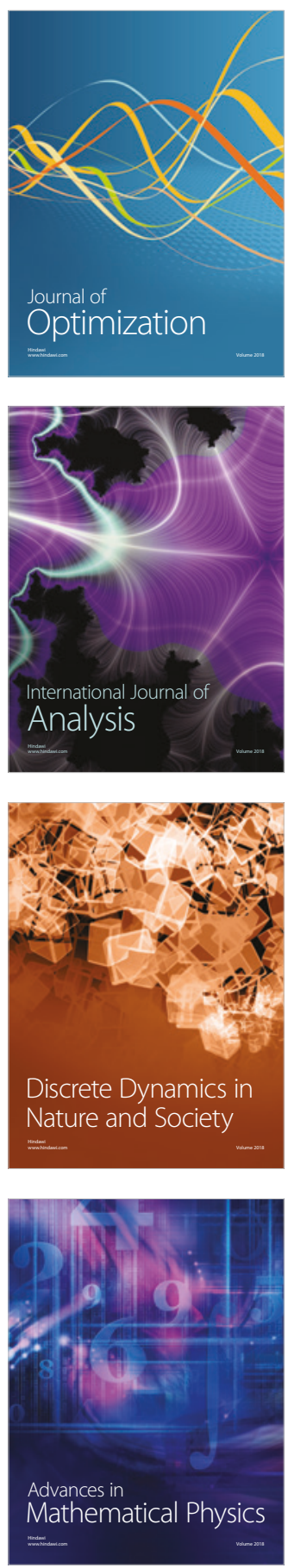